\title{
ANÁLISIS DE LOS EFECTOS DEL TRANSCURSO DEL TIEMPO EN EL TRIPLE SISTEMA DE RESPUESTA DE ANSIEDAD EN LAS SUPERVIVIENTES AL CÁNCER DE MAMA
}

\author{
ANALYSIS OF THE EFFECTS OF OVER TIME ON THE TRIPLE SYSTEM OF RESPONSE \\ OF ANXIETY ON BREAST CANCER SURVIVORS
}

\author{
Francisco García-Torres y Francisco J. Alós
}

Departamento de Psicología, Universidad de Córdoba/Instituto Maimónides de Investigación Biomédica de Córdoba (IMIBIC)/Hospital Universitario Reina Sofía de Córdoba (España)

Resumen

Objetivo: Evaluar la respuesta de ansiedad en tres sistemas (cognitiva, fisiológica y motora) en tres grupos de supervivientes al cáncer de mama y un grupo control para encontrar diferencias entre los grupos en los sistemas de ansiedad.

Método: 25 supervivientes al cáncer de mama fueron distribuidas en tres grupos según el tiempo desde que finalizaron el tratamiento (< de 5 años; > de 5 y $<10$ años; > de 10 años) y se incluyó un grupo control de 10 participantes de la población general sin historia de cáncer. Todas las participantes en el estudio completaron el Inventario de Situaciones y Respuestas de Ansiedad (ISRA).

Resultados: Los tres grupos de supervivientes obtuvieron valores más elevados que el grupo control en los tres sistemas de ansiedad sin llegar a ser significativos. En conjunto los resultados muestran valores elevados en ansiedad fisiológica y motora en los dos grupos más cercanos al final del tratamiento (< de 5 años; $>$ de 5 y $<10$ años). Además se observó que las puntuaciones en ansiedad cognitiva y motora aumentan en el segundo grupo comparado con el primero, disminuyendo posteriormente hasta obtener valores similares a los obtenidos por la población general.

Conclusiones: En general las supervivientes al cáncer de mama muestran una buena adaptación psicológica en términos de ansiedad, aun-

\section{Abstract}

Objective: Evaluate anxiety response in three systems (cognitive, physiological and motor) in three breast cancer survivors groups and a control group, to find differences between the groups in the anxiety systems.

Method: 25 breast cancer survivors were distributed into three groups according the time from the end of the treatment $(<$ than 5 years; $>$ than 5 and $<10$ years; $>$ than 10 years) and a control group of 10 participants from the general population without a cancer history were included. All study participants completed the Inventory of Anxiety Situations and Responses (ISRA).

Results: The three survivors groups obtained higher scores than the control group in the three anxiety systems, but not significant. Taken together the results shows high scores in physiologic and motor anxiety in the two groups closer to the end of the treatment $(<$ than 5 years; $>$ than 5 and $<\mathbf{1 0}$ years). Furthermore, it was observed that cognitive and motor anxiety systems values increases in the second group compared with the first, subsequently decreasing to obtain similar values with the general population.

Conclusions: In general breast cancer survivors shows a good psychological adjustment in terms of anxiety, although the data point out that is necessary taken into account the type of anxiety that may be more affected in each temporal time.

\section{Correspondencia:}


que los datos indican que es necesario tener en cuenta el tipo de ansiedad que puede estar más afectado en cada momento temporal.

Palabras clave: Cáncer, oncología, supervivientes, cáncer de mama, ansiedad cognitiva, ansiedad fisiológica, ansiedad motora.
Keywords: Cancer, oncology, survivors, breast cancer, cognitive anxiety, physiological anxiety, motor anxiety.

\section{INTRODUCCIÓN}

La presencia de ansiedad en las supervivientes al cáncer de mama ha sido documentada por diferentes autores en distintos países. A partir de los resultados obtenidos se observa que la ansiedad puede afectar a más de la mitad de las supervivientes evaluadas $^{(1-7)}$. En este grupo de pacientes existen determinadas características que se asocian con unos mayores niveles de ansiedad, como por ejemplo una menor edad, un nivel socioeconómico bajo, la falta de una relación íntima, haber recibido tratamiento psicológico previo a la enfermedad y tener una historia de experiencias vitales estresantes no relacionadas con el cáncer ${ }^{(2,8)}$. La ansiedad en las supervivientes al cáncer de mama se asocia con la presencia de depresión, alteraciones en el sueño, una menor calidad de vida, una mayor presencia de dolor y la fatiga ${ }^{(2,9-16)}$. Además, niveles elevados de ansiedad se relacionan con una peor imagen corporal y funcionamiento sexual, unas peores perspectivas sobre el futuro y la presencia de ideación suicida ${ }^{(17,18)}$.

La respuesta de ansiedad que se observa en las supervivientes se caracteriza por presentar aumentos después del diagnóstico de cáncer y estos niveles elevados se van reduciendo durante el tratamiento y el tiempo que sigue a la finalización del mismo, debido probablemente a la adaptación natural que sigue a la superación de una enfermedad grave como el cáncer $2,21-24)$ aunque por otro lado hay autores que observan que en las supervivientes al cáncer de mama, la ansiedad se mantiene elevada incluso años después del tratamiento ${ }^{(20,25-30),}$ sobre todo en aquellas con un menor nivel educativo y que han sido sometidas a radioterapia o quimioterapia ${ }^{(28)}$.

En la mayoría de los estudios que evalúan la ansiedad en este grupo de pacientes se aplican escalas que evalúan la ansiedad como rasgo-estado o aplicando instrumentos que miden la ansiedad excluyendo los aspectos somáticos ${ }^{(31,32)}$. A pesar de su utilidad, es posible que al aplicar este tipo de escalas queden sin recoger aspectos relevantes relacionados con la respuesta de ansiedad en las supervivientes. Debido a estas limitaciones, recientemente algunos autores han evaluado la ansiedad en las supervivientes al cáncer de mama partiendo de la concepción de la misma como una respuesta triple y disociada, incluyendo respuestas cognitivas, fisiológicas y motoras de ansiedad sin observar que existan diferencias entre un grupo de supervivientes y la población general cuando se comparan los resultados obtenidos por los dos grupos en los tres sistemas de ansiedad ${ }^{(33)}$. Sin embargo, es posible que estos resultados estén influenciados por la variabilidad en el tiempo desde el final del tratamiento de las supervivientes que participaron en dicho estudio, ya que como hemos visto anteriormente, la respuesta de ansiedad generalmente muestra disminuciones durante el tiempo que sigue al final del tratamiento. Por este motivo, el objetivo de este estudio es analizar la respuesta de ansiedad en las supervivientes al cáncer de mama de forma triple (cognitiva, fisiológica y motora) en tres grupos de supervivientes, agrupados en función del tiempo desde que finalizaron el tratamiento en tres momentos temporales: < de 5 años; > de 5 y $<$ de 10 
años y finalmente, $>$ de 10 años desde el final del tratamiento, para comprobar cómo responden los sistemas de ansiedad en los distintos momentos temporales. Además, los resultados obtenidos por las supervivientes en los tres sistemas de ansiedad en los diferentes grupos se compararán con los resultados obtenidos con un grupo control extraído de la población general.

\section{MÉTODO}

\section{Participantes}

Las participantes en el estudio fueron en total 25 mujeres que cumplían los criterios de inclusión y exclusión propuestos. Los criterios de inclusión para participar en el estudio fueron: a) haber recibido un diagnóstico de cáncer de mama; b) no estar actualmente bajo tratamiento; c) haber estado en los estadios I-III de la enfermedad. Los criterios de exclusión fueron: a) no haber recibido un diagnóstico de cáncer de mama o en caso de haberlo recibido, tener un segundo cáncer primario; b) estar actualmente en tratamiento; c) haber padecido una metástasis o recaída del cáncer primario. El tiempo desde el final del tratamiento osciló entre 1-21 años, con una media de 7,44 (DT=5,99). Para formar el grupo control se seleccionaron 10 mujeres de la población general que cumplieron con los criterios de inclusión: a) no haber padecido ningún tipo de cáncer; b) no tener un diagnóstico de otra enfermedad, y los criterios de exclusión: a) haber recibido un diagnóstico de cáncer; b) tener un diagnóstico de otra enfermedad.

\section{Instrumentos}

A todas las participantes en el estudio se les proporcionó un cuestionario que recogía información sobre aspectos de tipo sociodemográfico (edad, estado civil, educación y estado laboral), y en las super- vivientes al cáncer de mama se recogió información sobre el tratamiento al que habían sido sometidas y el tiempo desde la finalización del mismo. Posteriormente, se administró a cada participante una copia del Inventario de Situaciones de Respuestas y Ansiedad (ISRA) para que lo completasen. Este inventario se basa en el modelo multidimensional de la ansiedad y en el modelo de los tres sistemas de respuesta y recoge información sobre tres tipos de ansiedad de forma independiente: ansiedad cognitiva (pensamientos y sentimientos de preocupación, miedo o inseguridad), fisiológica (palpitaciones, taquicardias, sequedad bucal) y motora, que incluye respuestas de escape/ evitación como por ejemplo: fumar, beber y otro tipo de agitación motora como realizar movimientos repetitivos con las manos y los pies. Las puntuaciones directas obtenidas por los participantes en cada sistema se transforman en centiles utilizando los baremos proporcionados por los autores y que colocan la puntuación obtenida por el sujeto en relación a la población general, utilizando el baremo normal, y para conocer la severidad del grado de ansiedad se utiliza el baremo clínico. Este instrumento ha demostrado tener una adecuada consistencia interna y fiabilidad para su aplicación en población española ${ }^{(34)}$.

\section{Procedimiento}

Los autores del estudio contactaron con una asociación de pacientes de cáncer con sede en Córdoba (España), con la intención de solicitar su colaboración para la realización de la investigación. Después de recibir la aprobación del comité ético de dicha Asociación para llevar a cabo el estudio, se proporcionó a los profesionales que trabajaban directamente con las posibles participantes los criterios de inclusión y exclusión para participar en el estudio. Una vez que las participantes contactadas aceptaban participar en el estudio se les proporcio- 
naba un número de teléfono con el que podían ponerse en contacto con los investigadores y proceder a la firma del consentimiento informado y a la recogida de datos. En total 27 supervivientes cumplían con los criterios y fueron contactadas, sin embargo dos declinaron participar ya que no deseaban recordar su experiencia con el cáncer. Para formar el grupo control se contactó con 25 voluntarias de la Asociación de las cuáles todas aceptaron participar. De las 25 incluidas, se seleccionaron las 10 primeras participantes por orden de reclutamiento, cuyos datos se apartaron para su posterior análisis. A este grupo también se le proporcionó el mismo consentimiento informado, el cuál incluía información acerca de los objetivos del estudio, además este garantizaba la confidencialidad de los resultados e incluía de forma explícita la posibilidad de dejar de participar en el estudio en cualquier momento si así lo deseaban.

\section{Análisis estadísticos}

En un primer momento se llevaron a cabo los análisis descriptivos de las varia- bles sociodemográficas en cada grupo aplicando el análisis de frecuencias y la media con la desviación típica. Después se obtuvieron los estadísticos descriptivos de las medidas de ansiedad en cada grupo de forma independiente y para evaluar las posibles diferencias entre los grupos se aplicó la prueba $\mathrm{H}$ de Kruskal-Wallis debido al pequeño tamaño de las muestras que formaban los grupos y a su tamaño desigual. Los resultados se estimaron como significativos si $p \leq 0,05$.

\section{RESULTADOS}

En primer lugar, se dividió el grupo total de 25 supervivientes en grupos en función del tiempo desde la finalización del tratamiento. Así, se obtuvieron tres grupos: $<$ de 5 años; > de 5 años y < de 10 años; $>$ de 10 años, y 10 participantes al azar del grupo de voluntarias que formaron el grupo control. Los resultados descriptivos de las variables sociodemográficas a examen se describen en la tabla 1.

Posteriormente, realizamos el análisis descriptivo de las medidas de ansiedad de

\section{Tabla 1. Estadísticos descriptivos de las variables sociodemográficas en cada grupo examinado.}

\begin{tabular}{|c|c|c|c|c|c|}
\hline Grupo & $\begin{array}{c}\text { Edad } \\
\text { Media (DT) }\end{array}$ & E. Civil (\%) & Educación (\%) & E. Laboral (\%) & Tratamiento \\
\hline$<$ de 5 años & $56,20(9,35)$ & $\begin{array}{l}\text { Casada (80) } \\
\text { Viuda (10) } \\
\text { Soltera (10) } \\
\text { Separada (0) }\end{array}$ & $\begin{array}{l}\text { Primarios (80) } \\
\text { Secundarios (0) } \\
\text { Universitarios (20) }\end{array}$ & $\begin{array}{c}\text { Jubilada (60) } \\
\text { Desempleada (30) } \\
\text { Empleada (10) }\end{array}$ & $\begin{array}{c}\text { Mastectomía (90) } \\
\text { Quimioterapia (70) } \\
\text { Radioterapia (40) }\end{array}$ \\
\hline $\begin{array}{l}>5 \text { años y } \\
<10 \text { años }\end{array}$ & $56,85(5,45)$ & $\begin{array}{l}\text { Casada (72) } \\
\text { Viuda (28) } \\
\text { Soltera (0) } \\
\text { Separada (0) }\end{array}$ & $\begin{array}{l}\text { Primarios (58) } \\
\text { Secundarios (42) } \\
\text { Universitarios (0) }\end{array}$ & $\begin{array}{c}\text { Jubilada (43) } \\
\text { Desempleada (57) } \\
\text { Empleada (0) }\end{array}$ & $\begin{array}{c}\text { Mastectomía (86) } \\
\text { Quimioterapia (57) } \\
\text { Radioterapia (57) }\end{array}$ \\
\hline$>10$ años & $63,25(6,79)$ & $\begin{array}{l}\text { Casada }(62,5) \\
\text { Viuda }(12,5) \\
\text { Soltera }(12,5) \\
\text { Separada }(12,5)\end{array}$ & $\begin{array}{l}\text { Primarios }(87,5) \\
\text { Secundarios }(12,5) \\
\text { Universitarios }(0)\end{array}$ & $\begin{array}{c}\text { Jubilada (75) } \\
\text { Desempleada (25) } \\
\text { Empleada (0) }\end{array}$ & $\begin{array}{c}\text { Mastectomía (87) } \\
\text { Quimioterapia (75) } \\
\text { Radioterapia (37) }\end{array}$ \\
\hline Control & $55,40(9,55)$ & $\begin{array}{l}\text { Casada (80) } \\
\text { Viuda (20) } \\
\text { Soltera (0) } \\
\text { Separada (0) }\end{array}$ & $\begin{array}{l}\text { Primarios (50) } \\
\text { Secundarios (30) } \\
\text { Universitarios (20) }\end{array}$ & $\begin{array}{c}\text { Jubilada (20) } \\
\text { Desempleada (50) } \\
\text { Empleada (30) }\end{array}$ & \\
\hline
\end{tabular}


las que se recogió información en cada grupo por separado. Realizados los análisis para evaluar las diferencias entre los distintos grupos en las medidas de ansiedad no se observan diferencias significativas (ver la tabla 2). pervivientes que formaban el grupo podía influir en estos resultados ${ }^{(34)}$. Por este motivo, el objetivo que se planteó fue dividir a los supervivientes en grupos diferenciados desde el menor tiempo desde el final del tratamiento $(<$ de 5 años) pasando por un

\section{Tabla 2. Estadísticos descriptivos y comparaciones intergrupales de los sistemas de ansiedad.}

\begin{tabular}{cccc}
\hline Grupo & $\begin{array}{c}\text { A. Cognitiva } \\
\text { Media (DT) }\end{array}$ & $\begin{array}{c}\text { A. Fisiológica } \\
\text { Media (DT) }\end{array}$ & $\begin{array}{c}\text { A. Motora } \\
\text { Media (DT) }\end{array}$ \\
\hline < de $\mathbf{5}$ años $(\mathbf{n = 1 0})$ & $72,40(40,98)$ & $49,00(37,68)$ & $38,50(35,73)$ \\
> de $\mathbf{5}$ años y & $78,71(40,28)$ & $48,78(46,66)$ & $41,28(25,80)$ \\
< de 10 años $(\mathbf{n = 7 )}$ & $71,62(22,92)$ & $39,31(28,91)$ & $28,62(19,84)$ \\
> de 10 años (n=8) & $63,10(8,48)$ & $32,50(7,10)$ & $27,40(8,72)$ \\
Control $(\mathbf{n = 1 0 )}$ & $\chi^{2}(3)=0,80 ; p=0,84$ & $\chi^{2}(3)=1,22 ; p=0,74$ & $\chi^{2}(3)=1,22 ; p=0,74$ \\
\hline
\end{tabular}

\section{DISCUSIÓN}

La presencia de ansiedad en las supervivientes al cáncer de mama ha sido documentada por diferentes autores ${ }^{(1-7)}$. En líneas generales se observan aumentos en la ansiedad después de recibir el diagnóstico de cáncer y una disminución posterior durante el tiempo que sigue a la finalización de los tratamientos ${ }^{(2,19-24)}$. Sin embargo, en este grupo de pacientes la ansiedad habitualmente es tenida en cuenta como rasgo-estado o utilizando instrumentos que excluyen los síntomas somáticos lo que habitualmente conlleva una pérdida de información que puede ser relevante en el momento de evaluar con precisión la respuesta de ansiedad en los supervivientes ${ }^{(31-32)}$ aunque hay autores que han evaluado la ansiedad en las supervivientes al cáncer de mama de forma triple y disociada, observando que no existen diferencias entre este grupo y un control cuando se evalúa la ansiedad de esta forma. Sin embargo, la gran variabilidad observada en el tiempo desde el final del tratamiento de los su- periodo intermedio ( $>$ de 5 y $<$ de 10 años) y finalmente la supervivencia a largo plazo (> de 10 años) incorporando además un grupo control y además, evaluar la posible existencia de diferencias en las medidas de ansiedad (cognitiva, fisiológica y motora) entre los distintos grupos. Los resultados obtenidos van en la misma línea propuesta por los autores mencionados, ya que aunque los tres grupos de supervivientes obtienen valores más elevados que el grupo control no se observan diferencias significativas entre los grupos en las tres medidas de ansiedad, lo que parece avalar la idea de que después de finalizar el tratamiento del cáncer de mama, los niveles de ansiedad son similares a los obtenidos por la población general debido probablemente al proceso natural de adaptación que sigue a la superación de la enfermedad. Es necesario mencionar que cuando se analizan los resultados en conjunto, se observa que la ansiedad de tipo cognitivo se mantiene bastante estable en los tres grupos de supervivientes sin grandes variaciones, sin embargo, la ansiedad de tipo fisiológico y 
la ansiedad de tipo motor presentan valores elevados en los dos grupos más cercanos al final del tratamiento cuando se comparan con los grupos de $>$ de 10 años y el grupo control. Aunque como se comentó anteriormente no se observaron diferencias significativas entre los grupos, estos resultados resaltan la relevancia de tener en cuenta las respuestas de tipo fisiológico y motor que incluyen respuestas de escape/evitación y que pueden aparecer en las supervivientes al cáncer de mama hasta 10 años después del tratamiento. Estos resultados indican la presencia de ansiedad fisiológica y motora elevados en las supervivientes al cáncer de mama, los cuáles pueden relacionarse con la presencia de síntomas de trastorno de estrés postraumático que también se observan en este grupo de pacientes ${ }^{(35)}$. Si se analizan con detalle los datos obtenidos por las supervivientes en los distintos grupos, se observa un aumento de la ansiedad de tipo cognitivo y motor en el grupo de $>$ de 5 años y $<$ de 10 años, superando incluso a la ansiedad observada en estos dos sistemas en el grupo de menor tiempo desde el final del tratamiento $(<5$ años), manteniéndose la ansiedad fisiológica a niveles similares a los obtenidos en este último grupo. También se observan reducciones en los niveles de ansiedad a partir de los 10 años hasta obtener medidas similares a las del grupo control. Estos resultados pueden deberse a que en este momento temporal, alrededor de los 5 años desde el final del tratamiento, se producen cambios en las rutinas de seguimiento del cáncer que pasan a ser anuales, lo que puede favorecer la aparición de incertidumbre en las supervivientes y un aumento en la respuesta de ansiedad, respuesta que disminuye posteriormente si la enfermedad no vuelve a aparecer.

Finalmente, es necesario mencionar algunas limitaciones del presente estudio. En primer lugar los diferentes grupos que se obtienen al dividir el grupo de supervivientes son de tamaño limitado y esta circuns- tancia puede influir en los resultados obtenidos, por lo que sería aconsejable llevar a cabo estudios que con el mismo objetivo que incluyan grupos mayores y de igual tamaño. En segundo lugar, el instrumento utilizado no ha sido validado aún en pacientes o supervivientes al cáncer, y aunque muestra una buena validez convergente con el STAI, que es ampliamente utilizado en las supervivientes al cáncer de mama, es recomendable la validación del instrumento de forma más específica ${ }^{(32,35,37)}$. Además, para futuras investigaciones podría plantearse hacer una evaluación de las relaciones y el posible impacto que pueden tener estos sistemas de ansiedad sobre diferentes aspectos, como por ejemplo la calidad de vida de las supervivientes.

\section{CONCLUSIONES}

Los resultados obtenidos en su conjunto nos muestran una buena adaptación de las supervivientes al cáncer de mama una vez finalizado el tratamiento aunque los datos también nos llevan a pensar que es necesario tener en cuenta el tipo de ansiedad que puede presentarse en determinados momentos temporales con el objetivo de realizar unas intervenciones más ajustadas que alivien la presencia de estos síntomas en las supervivientes.

\section{REFERENCIAS BIBLIOGRÁFICAS}

1. Abu-Helalah M, Al-Hanagta M, Alshraideh $\mathrm{H}$, Abdulragi, N, Hijazeen, J. Quality of life and psychological well-being of breast cancer survivors in Jordan. Asian Pac J Cancer Prev 2014;15:5927-36. Doi: 10.7314/APJCP.2014.15.14.5927

2. Burguess C, Cornelius V, Love S, Graham J, Richards M, Ramírez, A. Depression and anxiety in women with early breast cancer: Five year observational cohort study. BMJ 2005; 330:702. Doi: 10.1136/ bmj.38343.670868.D3 
3. Braña-Marcos B, Carrera-Martínez D, De La Villa-Santoveña $M$, Vegas-Pardavilla E, Avanzas-Fernández S, Gracia-Corbato MT. Breast cancer survivors: Quality of life and prevailing diagnoses. Enferm Clín 2012;22:65-75. Doi:10.1016/j.enfcli.2011.10.004.

4. Brem S, Kumar NB. Management of treatment-related symptoms in patients with breast cancer. Clin J Oncol Nurs 2011;15:6371. Doi:10.1188/11.CJON.63-71.

5. Kenyon M, Mayer DK, Owens AK. Late and long-term effects of breast cancer treatment and surveillance management for the general practitioner. J Obstet Gynecol Neonatal Nurs 2014;43:382-98. Doi:10.1111/1552-6909.12300.

6. Mehnert A, Koch U. Psychological morbidity and health-related quality of life and its association with awareness, utilization, and need for psychosocial support in a cancer register-based sample of long-term breast cancer survivors. J Psychosom Res 2008;64, 38391. Doi:10.1016/j.jpsychores.2007.12.005.

7. Wang F, Liu J, Liu L, Wang F, Ma Z, Gao $D$, et al. The status and correlates of depression and anxiety among breast-cancer survivors in Eastern China: A population based, cross-sectional case-control study. BMC Public Health 2014;14:326. Doi: 10.1186/1471-2458-14-326.

8. Bohemer $U$, Glickman $M$, Winter $M$. Anxiety and depression in breast cancer survivors of different sexual orientations. J Consult Clin Psychol 2012;80:382-95. Doi: 10.1037/a0027494.

9. Aerts PD, De Vries J, Van der Steeg AF, Roukema JA. The relationships between morbidity after axillary surgery and long-term quality of life in breast cancer patients: The role of anxiety. Eur J Surg Oncol 2011;37:344-9. Doi:10.1016/j. ejso.2011.01.016.

10. Belfer I, Schreiber KL, Shaffer JR, Shnol H, Blaney K, Morando A, et al. Persistent postmastectomy pain in breast cancer survivors: Analysis of clinical, demographic, and psy- chosocial factors. J Pain 2013;14:1185-95. Doi:10.1016/j.jpain.2013.05.002.

11. Brunault P, Toledano A, Aguerre C, Suzanne I, Garaud P, Trzepidur-Edorn M, et al. Impact of late treatment-related radiotherapy toxicity, depression, and anxiety on quality of life in long-term breast cancer survivors. Bull Cancer 2012;99:58998. Doi: 10.1684/bdc.2012.1569.

12. Karakoyun-Celik O, Gorken I, Sahin S, Orcin E, Alanyali H, Kinay M. Depression and anxiety levels in woman under follow-up for breast cancer: Relationship to coping with cancer and quality of life. Med Oncol 2010;27:10813. Doi:10.1007/s12032-009-9181-4.

13. Keyzer-Dekker CM, De Vries J, Mertens MC, Roukema JA, Van der Steeg AF. Cancer or no cancer: The influence of trait anxiety and diagnosis on quality of life with breast cancer and benign disease: A prospective, longitudinal study. World J Surg 2013; 37:2140-7. Doi:10.1007/s00268-013-2088-4.

14. Lockefeer JP, De Vries J. What is the relationship between trait anxiety and depressive symptoms. Fatigue and low sleep quality following breast cancer surgery? Psychooncology 2013;22:1127-33. Doi:10.1002/pon.3115.

15. Pedersen AE, Sawatzky JA, Hack TF. The sequelae of anxiety in breast cancer: A human response to illness model. Oncol Nurs Forum 2010;37:469-75. Doi: 10.1188/10.ONF.469-475.

16. Tan XF, Xia F. Long-term fatigue in postoperative patients with breast cancer. Clin J Cancer Res 2014;26:12-6. Doi:10.3978/j. issn.1000-9604.2014.01.12.

17. Van Esch L, Roukema JA, Van der Steeg AF, De Vries J. Trait anxiety predicts diseasespecific health status in early-stage breast cancer patients. Qual Life Res 2011;20:86573. Doi:10.1007/s11136-010-9830-2.

18. Kim JM, Jang JE, Stewart R, Kim SY, Kim SW, Kang HJ, et al. Determinants of suicidal ideation in patients with breast cancer. Psychooncology 2013;22:2848-56. Doi: 10.1002/pon.3367. 
19. Bang SM, Park SH, Kang HG, Jue JI, Cho $\mathrm{IH}$, Yun $\mathrm{YH}$, et al. Changes in quality of life during palliative chemotherapy for solid cancer. Support Care Cancer 2005; 13:51521. Doi:10.1007/s00520-004-0708-0

20. Boyes AW, Girgis A, Zucca AC, Lecathelinais C. Anxiety and depression among long-term survivors of cancer in Australia: Results of a population-based survey. Med J Aust 2009;190:94-8.

21. Iconomou $G$, Mega $V$, Koutras $A$, Iconomou AV, Kalofonos HP. Prospective assessment of emotional distress, cognitive function, and quality of life in patients with cancer treated with chemotherapy. Cancer 2004;101:40411. Doi: 10.1002/cncr.20385

22. Jadoulle V, Rokbani L, Ogez D, Maccioni J, Lories G, Bruchon-Schweitzer M, et al. Coping and adapting to breast cancer: A six-month prospective study. Bull Cancer 2006; 93:67-72.

23. Khan NF, Ward AM, Watson E, Rose PW. Consulting and prescribing behavior for anxiety and depression in long-term survivors of cancer in the UK. Eur J Cancer 2010;46:333944. Doi:10.1016/j.ejca.2010.07.035.

24. Thompson J, Coleman R, Colwell B, Freeman J, Greenfield D, Holmes K, et al. Levels of distress in breast cancer survivors approaching discharge from routine hospital follow-up. Psychooncology 2013;22:1866-71. Doi:10.1002/ pon.3229. Doi:10.1002/pon.3229

25. Härtl K, Schennach R, Müller M, Engel $\mathrm{J}$, Reinecker $\mathrm{H}$, Sommer $\mathrm{H}$, et al. Quality of life, anxiety and oncological factors: A follow up study of breast cancer patients. Psychosomatics 2010;51:112-23. Doi:10.1176/appi.psy.51.2.112.

26. Hodkingson K, Butow P, Hunt GE, Pendlebury S, Hobbs KM, Wain, G. Breast cancer survivors' supportive care needs 2-10 years after diagnosis. Support Care Cancer 2007; 15:515-23. Doi 10.1007/s00520-006-0170-2

27. Kornblith $A B$, Ligibel J. Psychosocial and sexual functioning of survivors of breast cancer. Semin Oncol 2003;30:799-813. Doi:10.1053/j.seminoncol.2003.08.025

28. Schwarz R, Krauss O, Höckel M, Meyer A, Zenger $M$, Hinz $A$. The course of anxiety and depression in patients with breast cancer and gynaechological cancer. Breast Care (Basel) 2008;3:417-22. Doi: 10.1159/000177654

29. Vahdannia M, Omidvari S, Montazeri A. What do predict anxiety and depression in breast cancer patients? A follow-up study. Soc Psychiatry Psychiatr Epidemiol 2010;45:35561 Doi:10.1007/s00127-009-0068-7.

30. Yildirim NK, Ozkan M, Ozkan S, Ozcinar B, Guler SA, Ozmen V. The anxiety, depression and quality of life of breast cancer patients before and after treatment: The results of one year prospective study. Noropsikiyatri Arsivi 2009;46:175-81.

31. Klein D, Mercier M, Abeilard E, Puyraveau M, Danzon A, Dalstein V, et al. Long-Term quality of life after breast cancer: A French registry-based controlled study. Breast Cancer Res Treat 2011;129:125-34. Doi: 10.1007/s10549-011-1408-3.

32. Alexander S, Palmer C, Stone PC. Evaluation of screening instruments for depression and anxiety in breast cancer survivors. Breast Cancer Res Treat 2010;122:573-8. Doi: 10.1007/s10549-009-0669-6.

33. Garcia-Torres F, Alós FJ. Anxiety in common situations of everyday life in breast cancer survivors. Psychooncology 2014;23:953-5. Doi:10.1002/pon.3548.

34. Miguel-Tobal JJ, Cano-Vindel AR. Inventario de Situaciones y Respuestas de Ansiedad. Manual. Madrid: TEA, 1988.

35. Koutrouli N, Anagnostopoulos F, Potamianos G. Postraumatic stress disorder and posttraumatic growth in breast cancer patients: A systematic review. Women Health 2012;52:503-516. Doi:10.1080/03630242.2 012.679337 .

36. Lebel S, Beattie S, Arès I, Bielajew C. Young and worried: Age and fear of recurrence in breast cancer survivors. Health Psychol 2013;32:695-705. Doi:10.1037/a0030186. 